\title{
Veinticinco años del Grupo de Comunicación La Región (1985-2010)
}

Recibido: $26 / 10 / 2012$

\author{
Mercedes ROMÁN PORTAS \\ Universidad de Vigo \\ mroman@uvigo.es \\ Beatriz LlOVES SOBRADO \\ Universidad de Vigo \\ beatrichecom@gmail.com
}

Aceptado: 23/01/2013

\section{Resumen}

Este artículo tiene como objetivo explicar los medios de comunicación del Grupo La Región desde el año 1985 a 2010. El primer número de La Región salió a la calle el 15 de febrero de 1910. Este diario es el origen y matriz del Grupo. Años después, en 1966 nació La Región Internacional. En los últimos 25 años el Grupo se completó con Atlántico Diario, Telecies, Telemiño y Santiago T.V. La elección de este periodo tiene como finalidad, por un lado, exponer los cambios producidos desde 1985 a 2010 y dar continuidad a las tesis de Edelmiro Valladares (Valladares: 1996); y por otro, trata de explicar la permanencia e implicación del diario La Región tanto Orense como en sus localidades.

Palabras clave: historia, prensa, grupo comunicación, televisión local.

\section{Twenty-Five Years of the Communication Group La Región (1985-2010)}

\begin{abstract}
This article is aimed at explaining the means of Communications of the Grupo La Región from the year 1985 to 2010 . The first issue of La Región, which is the origin and matrix of the group, came out on $15^{\text {th }}$ February 1910. Years later, in 1966 La Región Internacional was founded. In the last twenty-five years, Altlántico Diario, Telecies, Telemiño and Santiago T.V. were added to this group. On the one hand, the choice of this period is to exhibit the changes which were brought about from 1985 to 2010 and to provide continuity to the Thesis of Edelmiro Valladares; and on the other hand, to try and explain the permanence and implication of La Región newspaper in Orense as well as other localities.
\end{abstract}

Keywords: history, newspapers, group communication, local television.

\section{Referencia normalizada}

ROMÁN PORTAS, Mercedes y LLOVES SOBRADO, Beatriz (2013): "Veinticinco años del Grupo de Comunicación La Región (1985-2010)". Estudios sobre el Mensaje Periodístico. Vol. 19, Núm. especial marzo, págs.: 409-417. Madrid, Servicio de Publicaciones de la Universidad Complutense.

Sumario: 1. Introducción. 2. Metodología y fuentes. 3. Medios de comunicación del Grupo La Región; 3.1. La Región; 3.2. La Región Internacional; 3.3. Atlántico Diario; 3.4. Telemiño; 3.5. Telecies; 3.6. Santiago T.V. 4. Conclusiones. 5. Referencias bibliográficas.

\section{Introducción}

En el año 2010, el diario La Región de Ourense cumplió 100 años. Los primeros 75 años de su historia fueron estudiados por Edelmiro Valladares. Con este trabajo queremos presentar la evolución de este diario gallego y la conversión de la empresa en grupo multimedia de comunicación, desde el año 1985 al año 2010.

Los medios de comunicación que forman parte del Grupo son, por un lado, los medios impresos: el diario La Región (1910), La Región Internacional -desde 1966- y Atlántico Diario (1987), y por otro los medios audiovisuales; Telemiño (1999), Telecíes (2008) y Santiago TV que pertenece a La Región desde 2010. 
El objetivo de este estudio consiste en intentar explicar cuáles son las causas de la permanencia de La Región, diario centenario de la ciudad de Ourense. De los nueve diarios que existen en Galicia, La Región ocupa la sexta posición. Su tirada y difusión se caracterizaron durante estos veinticinco años por ser bastante estables y bajas.

La investigación parte de la hipótesis de que La Región es un diario que sigue vivo por su implicación en Ourense y en su provincia y por la necesidad que de él tienen las instituciones y la sociedad. A lo largo del trabajo se estudiarán las estrategias empresariales llevadas a cabo y las ayudas y subvenciones recibidas, a fin de conocer en qué medida se mantiene más con estas ayudas que con sus propios ingresos y cómo ha sobrevivido a la crisis que se hizo manifiesta a partir de 2007.

Estudiaremos el nacimiento de cada uno de los medios del grupo tanto en el sector de la prensa escrita como en el sector audiovisual. Analizaremos por tanto su participación en la televisión local, y las licencias que recibió en el concurso de Televisión digital terrestre local.

\section{Metodología y fuentes}

La metodología de este estudio se basó en la utilización y análisis de fuentes primarias y secundarias.

Las fuentes primarias utilizadas han sido las siguientes: los números publicados de La Región (1985-2010), los especiales (de 1996 y 2010), los libros de Actas del Consejo de Administración (1985-2010), y las Memorias de Cuentas Anuales e Informes de Gestión de La Región S.A. (1992-diciembre 2009).

Las fuentes bibliográficas (secundarias) son principalmente los trabajos realizados sobre la empresa de La Región y de otras empresas periodísticas.

El Grupo La Región es una empresa familiar. Casi el 98\% pertenece a la familia Outeiriño y tan sólo el 2,37\% pertenece a otras empresas y particulares.

\section{Medios de comunicación del Grupo La Región}

\subsection{La Región}

A lo largo del último siglo, el diario La Región se convirtió en el periódico de los orensanos. Supone el 80 por ciento del mercado de la prensa diaria en la provincia y el 90 por ciento del mercado publicitario local, realizando el 70 por ciento de sus ventas de publicidad en Ourense ${ }^{1}$.

En estos veinticinco años La Región empezó a publicar suplementos que se distribuyen conjuntamente con el periódico. El denominador de común de todos ellos es informar sobre la ciudad de Ourense y sus localidades. Actualmente el diario cuenta con ocho suplementos y con una revista mensual Auria.

En cuanto a las cifras de tirada y difusión del diario La Región durante el periodo estudiado (1985-2010), son estables y bajas. Si se hace una comparativa con el resto de periódicos de Galicia que están sometidos a la OJD, La Región ocupa el sexto lugar en relación con los nueve diarios gallegos.

Los años de mayor tirada y difusión se dieron en la década de los noventa y el inicio del siglo XXI superando la tirada de los 15.000 ejemplares. Las cifras más bajas

${ }^{1}$ Especial 100 años de La Región 1910-2010, pág. 222 
tuvieron lugar a finales de la década de los ochenta y a partir de 2004 hasta la actualidad, donde la tirada no alcanza los 14.000 ejemplares. A partir de 2005 la cifra vuelve a descender llegando al año 2010 con los datos más bajos de tirada y difusión de los últimos 25 años, con una tirada de 13.164 ejemplares y una difusión de 11.105

Durante estos veinticinco años el diario La Región estuvo dirigida por cuatro directores. Alejandro Outeiriño Rodríguez desde 1968 hasta 1989. Francisco Campos Freire, desde 1989 hasta 1990. Alfonso Sánchez Izquierdo desde 1990 hasta 2008 y Xóse Pastoriza Martínez, desde 2008 hasta la actualidad.

\subsection{La Región Internacional}

El primer número de este periódico salió a la calle el 1 de noviembre de 1966. Al inicio se hicieron dos ediciones: La Región edición aérea para Europa y La Región, edición aérea para América. Fue en el año 1981 cuando ambas publicaciones se funden en una sola cabecera con el nombre de La Región Internacional, aunque seguían manteniendo el subtítulo de Edición Europa y Edición América respectivamente hasta el año 1993 (Vázquez, 2000: 69). En este año, y hasta el término de este estudio en el 2010, se comienza a editar un único periódico, La Región Internacional, tanto para Europa como para América.

Cuando se elaboraban las dos ediciones -La Región edición aérea para Europa y La Región, edición aérea para América- se empleaba el papel denominado "biblia" porque pesaba poco y los costes eran más reducidos. A partir de 1994 el papel que se emplea es normal porque el papel "biblia" pasó a ser más caro y además daba problemas en la rotativa a la hora de ser impreso ya que era muy fino.

Al principio, se hacían dos tiradas por semana -martes y jueves- pero desde el 2007 la tirada es quincenal por motivos económicos, así se reflejaba en el Acta del Consejo de Administración, del 6 de agosto de 2007:

"Necesidad de adoptar la estructura de la Edición Internacional a los tiempos actuales, sobre todo, la posibilidad de reducir los dos principales costes, el papel y la distribución -correos- que ya se ha reducido de bisemanal a semanal. Se pretende sustituir el soporte actual en papel por un soporte a través de Internet"'.

Actualmente se envían los ejemplares de La Región Internacional a una distribuidora suiza llamada Swiss-Post que se encuentra en Madrid.

La estructura del periódico cuenta con un número de páginas que oscila entre 40 y 56. La principal información que contiene hace referencia a noticias relacionadas con los españoles en el mundo, con informaciones dedicadas a las comunidades españolas y a noticias generales de España.

La Región Internacional también realiza reportajes donde se tratan temas que se caracterizan por tratar de aspectos que tienen relación con lo que a los residentes en el exterior le pueda afectar. También hacen referencia a la economía -como reformas de las pensiones-, a los deportes y sociedad, que tratan siempre de temas relacionados con el exterior y la emigración.

2 Acta del Consejo de Administración de La Región S.A., 06-VIII-2007. 
En La Región Internacional se publicaron estudios sobre la realidad de la emigración en los años 1978, 1984 y 2000. El de este último año fue el de mayor envergadura y extensión y fue dirigido por Amando de Miguel que elaboró un estudio sociológico sobre la emigración con el título de Los emigrantes españoles.

La Región Internacional es gratuita para todos los emigrantes. También es la única publicación destinada a los emigrantes sometida a los controles de difusión de la OJD.

Los datos de 2010 muestran que este diario está presente en dieciocho países de América con un total de 2.246 ejemplares. Los países con mayor número de lectores en América de La Región Internacional son: Argentina -porque es donde hay más españoles-, con 945 ejemplares; Venezuela -152 ejemplares-; EE.UU -121 ejemplares; Méjico -306 ejemplares- y Uruguay -128 ejemplares-.

También se distribuye en otros cuatro destinos repartidos por el resto del mundo: Australia -11 ejemplares-, Marruecos - 3 ejemplares-, China -5 ejemplares- y Kenia 5 ejemplares-, lo que suma un total de 24 ejemplares.

Con respecto a Europa, el total de ejemplares distribuidos es de 4.340. El mayor número de lectores se produce en Suiza -489 ejemplares-; Alemania -723 ejemplares; Francia -611 ejemplares-; Holanda -544 ejemplares-; y Bélgica -102 ejemplares-. Estos lugares fueron los principales destinos de los emigrantes orensanos.

Los principales lugares de distribución de La Región Internacional en el territorio español son: Ourense y provincia -211-; Coruña -188-; resto de España -176-; Oviedo -83- y en Madrid -55-.

La distribución es notablemente menor en las ciudades de Huesca -3 ejemplaresy Cáceres -5 ejemplares-.

\subsection{Atlántico Diario}

En 1986, un centenar de inversores, la mayoría de Vigo, constituyeron la Sociedad Anónima Rías Baixas Comunicación. El 71,14\% de esta empresa es de La Región S.A., por tanto forma parte del Grupo La Región.

En su creación, fue presidida por el cirujano Juan Mosquera Luengo y José Luis Outeiriño Rodríguez, como consejero-delegado. De este proyecto nació al año siguiente Atlántico Diario, que se presentaba como "diario independiente de Galicia" ${ }^{3}$. Su primer número salió a la calle el 14 de junio de 1987.

Atlántico Diario es un periódico de Vigo; no obstante su impresión se hace en la rotativa del diario La Región, en el Polígono de San Ciprián de Viñas -Ourense. Su edición digital se puso en marcha en el año 2009.

Varios de los suplementos que se publican con Atlántico Diario se publican también en La Región, para ahorrar gastos y abaratar costes.

La empresa barcelonesa denominada Información Técnica y Científica S.L. ${ }^{4}-$ Infortécnica- realizó un estudio profundo sobre la audiencia de Atlántico Diario en el año

3 http://es.wikipedia.org/wiki/Atl\%C3\%A1ntico_Diario

4 Infortécnica es una empresa barcelonesa que se creó en el año 2000. Su trabajo consiste en la realización y certificación de estudios de audiencia de medios de comunicación en el área específica de difusión del medio, ya sea un municipio, una región, un país o todo un conti- 
2003 y es el más reciente realizado por la empresa. El estudio se hizo con una muestra de 710 personas. Con 455 entrevistas en la ciudad de Vigo y 255 entrevistas en poblaciones de Cangas, Moaña, Porriño, Mos, Nigrán, Baiona, A Guardia y Tui. El tipo de entrevista fue telefónica, aleatoria y de distribución proporcional a la extensión de la población.

Los resultados de este estudio muestran que Atlántico Diario es conocido por el $88,9 \%$ de los lectores habituales de prensa de Vigo y localidades de su entorno.

El $15,8 \%$ de la población a la que se le hizo el estudio lee habitualmente Atlántico Diario. Un 53,3\% lo lee ocasionalmente. Un $10,3 \%$ lo dejó de leer y un $20,7 \%$ no lo leyó nunca.

Un $41,9 \%$ de la población dedica a leer el diario entre 15 y 30 minutos. Sólo un $3,4 \%$ le dedica más de una hora. El lugar donde más se lee Atlántico Diario es en el bar o cafetería -45,5\%-. De ahí que el 57,6\% de la población no compra el periódico frente a un $42,4 \%$ que si lo hace. Los días que más se lee es entre lunes y vienes -55\%, descendiendo a un 7\% los domingos. El perfil del diario es definido por casi el $90 \%$ de los entrevistados como objetivo y ameno. El 46,2\% de las personas que no leen Atlántico Diario es porque prefieren otros periódicos.

La valoración general es positiva por la el conjunto de la población. La valoración más alta hace referencia a la calidad de la impresión -con una puntuación de 7,36- y los suplementos - con una nota de 7,35-. Lo menos valorado es la publicidad -con un 6,23-

Atlántico Diario, alcanza un $8,9 \%$ de cuota de audiencia, entre los periódicos. El 91,10\% lo ocupan: ABC, El Mundo, El País, Faro de Vigo y La Voz de Galicia.

Un $42,4 \%$ de los lectores afirman comprar el periódico por lo menos de forma ocasional.

En el año 2010 Atlántico Diario es el periódico con las cifras más bajas del conjunto de la prensa gallega. Su tirada es de $5.144^{5}$ y los lectores diarios de $19.000^{6}$.

Una vez definidos los medios de comunicación del Grupo La Región en el área impresa, se muestra a continuación la posición de estos diarios dentro de la prensa regional gallega, en el año 2010, en cuanto a su tirada y difusión (elaboración propia):

\begin{tabular}{|l|c|c|c|}
\hline Periódico & Tirada $^{\mathbf{7}}$ & Difusión $^{\mathbf{8}}$ & Lectores/día $^{\mathbf{9}}$ \\
\hline La Voz de Galicia & 111.518 & 97.016 & 585.000 \\
\hline Faro de Vigo & 44.770 & 38.004 & 268.000 \\
\hline Correo Gallego & 27.477 & 22.735 & 79.000 \\
\hline El Progreso & 18.307 & 15.258 & 93.000 \\
\hline El Ideal Gallego & 13.705 & 10.803 & 18.000 \\
\hline La Región & 13.164 & 11.105 & 79.000 \\
\hline
\end{tabular}

nente. Los estudios se realizan mediante entrevistas telefónicas, con un nivel de confianza del $95,5 \%$.

${ }^{5}$ OJD 2010.

${ }^{6}$ EGM 2010.

7 OJD -Oficina de Justificación de Medios-, 2010.

8 OJD -Oficina de Justificación de Medios-, 2010.

9 EGM -Estudio General de Medios- febrero/noviembre, 2010. 


\begin{tabular}{|l|l|l|l|}
\hline Diario de Pontevedra & 7.999 & 6.888 & 44.000 \\
\hline La Opinión & 7.430 & 5.616 & 35.000 \\
\hline Atlántico Diario & 5.144 & 4.014 & 19.000 \\
\hline
\end{tabular}

Los datos más recientes de audiencia, hacen referencia a un estudio realizado por la empresa Avante Marketing\&Media del año 2012. Según estos datos ${ }^{10}$, el ranking de los diarios gallegos es el siguiente:

Los medios en Galicia. Ránking diarios.

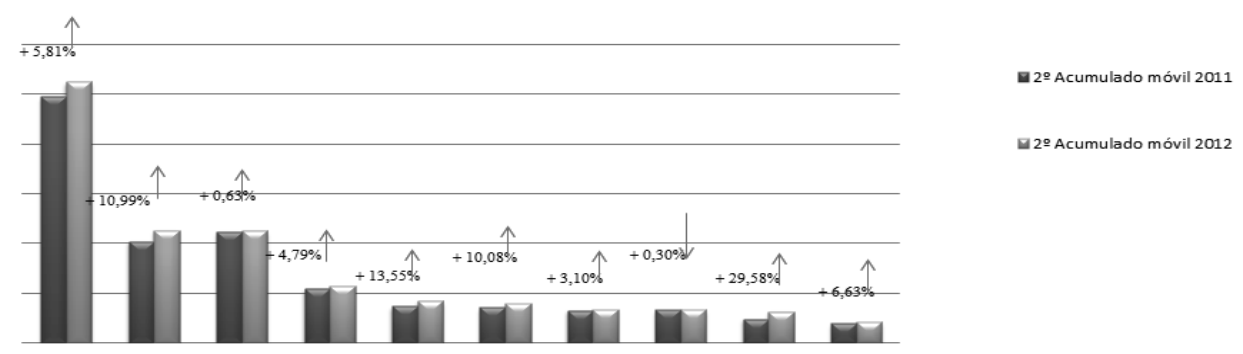

Figura 1 Fuente: EGM. $2^{\circ}$ Acumulado Móvil 2011 y $2^{\circ}$ Acumulado Móvil 2012

La Región, pese a que ha subido en su número de lectores, en un 3,10\% sigue ocupando una posición baja dentro de la prensa gallega.

\subsection{Telemiño}

Telemiño ${ }^{11}$ es la televisión local de Orense y forma parte del Grupo La Región. Antes de la primera emisión regular, Telemiño realizó tres retransmisiones en directo: la primera desde el Teatro Principal con motivo de la inauguración de la temporada de $E l$ Foro La Región. También lo hizo en otra ocasión desde el Aula de Cultura Caixanova y desde el parque Tecnológico de Galicia, con motivo de la inauguración de la sede de la empresa de telecomunicaciones Egatel.

Empezó a emitir el 31 de mayo de 1999. Únicamente emitía dos programas: un informativo de diez minutos y el programa Largar y Largar. Telemiño opera en la ciudad de Orense y sus alrededores donde ha alcanzado una cuota de 48.000 espectadores según datos del EGM ${ }^{12}$. A partir de octubre de 1999 se amplía la producción propia con la aparición de nuevos programas.

Los programas de producción propia de Telemiño son o han sido: Punto Crítico programa de debate-, Boca a Boca, Sin cita previa; Agora Ourense-Ourense Actual, diferentes etapas; La tertulia, Diálogos entre dos, Teleniños, Las Cuentas Claras,

${ }^{10}$ Los Medios en Galicia. Informe sobre el consumo de medios según EGM y Kantar Media, septiembre 2012.

$11 \mathrm{http}: / /$ es.wikipedia.org

12 EGM -2 ${ }^{\mathrm{a}}$ ola 2009-. 
Xente Nova, Xente Maior, Sen Barreiras, El rompecabezas, Código 168, Con el sexo, no te cortes, Entrevistas con Ourense, Dos de cine, Paralelas, Más Entrevistas, Nova Ourense, Cousas de comer, Vamos como motos, Galas de inauguración del Festival de Cine de Ourense, directos en jornadas electorales, directos en eventos deportivos como la carrera de San Martiño o el Rally de Ourense, resúmenes de los plenos celebrados en el Ayuntamiento y la Diputación Provincial, etc.

Los mayores esfuerzos de la emisora se han concentrado en la realización de directos y programas especiales en grandes eventos como el Rallye de Ourense, las galas de inauguración del Festival Internacional de Cine Independiente de Ourense, las jornadas electorales, ciertas retransmisiones deportivas, los plenos del Ayuntamiento, o las ferias de la Fundación ourensana de Exposiciones Expourense.

En julio de 2006 la Xunta de Galicia adjudicó las emisoras digitales de cobertura local. Telemiño recibió cinco licencias de TDT-L (Román, 2008: 48-50) en las demarcaciones de Orense, O Barco de Valdeorras, Carballiño, Verín y Monforte.

$\mathrm{Al}$ igual que con Atlántico Diario, la empresa Infortécnica, hizo dos estudios de audiencia sobre Telemiño. Uno en el 2001 y otro en el 2003.

En el estudio más actual, el de 2003, la muestra fue de 712 personas, mayores de edad, de Orense. El tipo de entrevista, al igual que en Atlántico Diario, fue telefónica, aleatoria y de distribución proporcional a la extensión de la población.

Si se comparan los estudios realizados en el 2001 y 2003. Telemiño ha pasado de tener un tiempo medio de sintonización de seis minutos diarios a doce, alcanzando una cuota de audiencia share del 6\% del conjunto de emisoras que se reciben en Ourense capital. También se han incrementado los índices de conocimiento del canal de televisión local.

Casi toda la población de Ourense, un 93,5\%, conoce Telemiño y un $80 \%$ la tiene presintonizada. La mitad de la población la ve todos los días, es decir alrededor de 60.000 personas. Por tanto, la cuota -GRP's- de telespectadores que le corresponde es de 7.200 personas sólo en la capital, superando 8.000 en su área de difusión.

El momento de mayor audiencia está comprendido entre las 21 y 23 horas, con una audiencia conjunta de 4.500 personas.

Los programas que más le interesan a las personas que ven esta televisión son los relacionados con las noticias e informativos locales $43 \%$, seguido de las entrevistas y debates $18,1 \%$. Los menos valorados por los telespectadores con los magazines $4,6 \%$.

\subsection{Telecies}

Telecíes ${ }^{13}$ es una televisión local que emite en Vigo. Pertenece a la empresa Rías Baixas Comunicación S.A. que, como ya se ha dicho, forma parte del Grupo La Región.

Las primeras emisiones fueron en julio de 2006, cuando la Xunta de Galicia adjudicó las emisoras digitales de cobertura local. En este sentido, Telecíes recibió dos licencias de TDT-L en las demarcaciones de Vigo y Ponteareas.

Los días 8, 9 y 10 de febrero de 2008 realizó su primera emisión, así lo comunicaba el diario Atlántico Diario:

${ }^{13} \mathrm{http}: / /$ es.wikipedia.org 
“Telecíes, la nueva televisión de Vigo, emitirá en directo por el canal 49 UHF. La televisión local de Atlántico inicia su primera fase de pruebas retransmitiendo los tres días. La emisión se podrá captar en el canal 49 UHF Domaio y también se retransmitirá la señal en Orense a través de Telemiño, perteneciente al mismo grupo de Atlántico, Grupo La Región [...]. El gran despliegue de medios permitirá que hasta TeleDeporte pueda captar la señal emitida por Telecíes. Una unidad móvil, una lanzadera satélite y una grúa de 12 metros de largo darán al espectáculo la magnitud y atención que merece [...]"14.

La retransmisión, en directo, se hizo desde el IFEVI, de la Rolex FEI World Cup Jumping, que se celebraba en Vigo por séptimo año consecutivo presentada por Laura Vázquez y Soledad Parral y con los comentarios de David Ferrer y María Teresa Jiménez, profesora de salto en el Centro Ecuestre de la Diputación de Orense.

\subsection{Santiago $T V$}

Es el medio de comunicación del Grupo La Región más reciente. Nace el 10 de noviembre de 2010. El contrato de gestión con Santiago TV se realizó el 1 de noviembre de ese año.

Su objeto social es la distribución, comercialización, exportación e importación de publicaciones periódicas escritas y elementos promocionales. Comercialización de publicidad y prensa. También lleva a cabo la gestión indirecta o a través de alquiler, subcontrata de cualquier concesión administrativa en relación con este aspecto, del servicio de Televisión Local por ondas terrestres. Su principal accionista es Ouro S.A. con el $100 \%$ de capital y el administrador único es Oscar Outeiriño Vila ${ }^{15}$.

\section{Conclusiones}

1. Durante el periodo estudiado (1985-2010) el Grupo La Región fue creciendo hasta configurarse en el año 2010 con seis medios de comunicación. Tres en el ámbito impreso: La Región (1910), La Región Internacional (1966) y Atlántico Diario (1987); y tres en el audiovisual: Telemiño (1999), Telecies (2008) y Santiago T.V. (2010).

2. EL Grupo La Región es una empresa totalmente familiar. Casi el $98 \%$ pertenece a la familia Outeiriño y tan sólo el $2,37 \%$ pertenece a otras empresas y particulares.

3. El diario La Región es el origen de este Grupo cuyo nacimiento fue en 1910. En el año 2010 pasa a formar parte de la prensa centenaria. La Región se caracterizó por tener unas cifras de tirada y difusión estables y bajas durante estos veinticinco años.

4. La mayor distribución de La Región se realiza en Ourense y en las localidades de Verín, Xinzo da Limia y Carballiño.

5. De los nueve diarios que hay dentro de la prensa gallega, La Región ocupa el sexto lugar y Atlántico Diario, otro de los medios del Grupo, la última posición, la novena.

14 www.atlantico.net, 08-II-2008

15 Registro Mercantil, 2010 
6. En julio de 2006 la Xunta de Galicia Adjudicó las licencias de TDT y Ourense recibió cinco licencias en las demarcaciones de Orense, O Barco de Valdeorras, Carballiño, Verín y Monforte.

\section{Referencias bibliográficas}

ROMÁN PORTAS, Mercedes (2008): “Televisión Digital Terrestre Local en Galicia”. Revista Ámbitos, $\mathrm{n}^{\circ} 17$.

VALLADARES FERNÁNDEZ, Edelmiro (1996): Historia de una empresa periodística gallega: La Región. Tesis doctoral. Madrid, Editorial Universidad Complutense.

VÁZQUEZ ÁLVAREZ. María Belén (2000): La Región Internacional: el periódico de los españoles en el mundo. Facultad de Ciencias Sociales y de la Comunicación, Pontevedra-Libros del Registro Mercantil de la empresa La Región S.A. (19852010).

\section{Mercedes ROMÁN PORTAS}

Universidad de Vigo

Profesora titular del área de periodismo

mroman@uvigo.es

\section{Beatriz LLOVES SOBRADO}

Universidad de Vigo

beatrichecom@gmail.com 